\title{
ASME B16.9 Piping Components: An Analysis of the Critical Dimensions
}

\author{
Gwen Anderson ${ }^{1}$ Ida Helene Gjerde ${ }^{1}$ Sondre Luca Helgesen ${ }^{2}$ Mladen Jemenica ${ }^{1}$ Rajan Kumar Thapa ${ }^{1}$ \\ ${ }^{1}$ Department of Process, Energy and Environmental Technology, University of South-Eastern Norway, Norway \\ gwendolyn.anderson@outlook.com; idahbgjerdelgmail.com; \{mladen.jecmenica, \\ rajan.k. thapa\} eusn.no \\ ${ }^{2}$ Stressman Engineering AS, Norway slh@stressman. no
}

\begin{abstract}
Pipe components such as elbow, tees, and weldolets are produced at various places in the world. Although the component is produced in accordance with standard ASME (American Society of Mechanical Engineers) B16.9, measurements show variation in geometry. In piping component design, the geometries are assumed as perfect in accordance with the producer's specifications. This is a conservative approach in design that results in incorrect dimensioning of the components. Moreover, components made of duplex materials can have failure due to Hydrogen Induced Cracking (HISC) especially in subsea installations.

In this article, a series of measurements were taken for ASME B16.9 long radius elbows, utilizing an ultrasonic thickness gauge. The results are compared with the nominal straight pipe dimensions in ASME. The deviation is taken as a basis for Finite Element analysis.

A model of the elbow is developed using SolidWorks and simulated using ANSYS. The results compare the strength of the pipe fittings against their straight pipe counterparts with regard to internal pressure. This gives an overview of the deviations and utilization based on the measurements taken against the standard ASME B16.9 geometry.
\end{abstract}

Keywords: $\quad$ ASMEB16.9, piping, pipe bends, pipe elbows, FEM, standard components, ultrasonic thickness gauge

\section{Introduction}

HISC is a form of cracking caused due to high concentration of hydrogen in materials which leads to high amounts of stress at a concentrated area and can cause the pipe component to burst. This occurs when hydrogen diffuses into the material structure and changes the material's microstructure. The cracking tends to form parallel to the surface or the same direction as hoop stress. The structure will not immediately give in, but over time when being exposed to both strain and tensile stress, the pipe will suffer from internal cracking (Inspectioneering, 2020).

Pipe components used in the subsea industry are frequently exposed to HISC which weakens the component. Guidelines for avoidance of HISC is given in DNVGL-RP-F112 (Veritas, 2018) whilst ASME 16.9 (Engineers, ASME B16.9, 2012) requires the structural integrity for standard butt-welded components. Are all pipe components according to standard, and is the standard comprehensive enough? This question gives a background for why reevaluating ASME B 16.9 is relevant. The Standard requires the pipe components to be of equivalent strength to its straight pipe counterpart. It is questioned if it is possible to confirm this statement, say something about the lower bound values for analysis and map eventual critical dimensions.

Details of the standard pipe bend components are evaluated by measuring the wall thickness using an ultrasonic thickness gauge. The data collected is evaluated both statistically as well as modeled in SOLIDWORKS and simulated using the Finite Element Method (FEM).

\section{Pipe measurements}

This article was started by mapping of critical dimensions of pipe components such as bends. Data was collected during a four-day visit to Scandinavian Flanges and Fittings (SFF) in Sandnes, Norway. The goal is to define a limitation for wall thickness in the form of a specific percentage or value.

\subsection{Measurement equipment}

The equipment used to take measurements was the Ultrasonic thickness gauge model MG26 from Panametrics-NDT. This gauge has a thickness range of $0.05 \mathrm{~mm}$ to $508 \mathrm{~mm}$ and a resolution of 10 micrometers. Ultrasonic rays do not have a good connection through the air, so the liquid gel is required to make proper contact with the component surface. The soap, Zalo was used as the gel. At the time of measurement, materials were not temperature controlled. According to Yr.no, temperatures in Sandnes, were between $2-8^{\circ} \mathrm{C}$. The density of the material's grain spacing effects how the ultrasonic rays penetrates the material. Therefore, some materials are more difficult to measure than others.

\subsection{Measurement locations}

To have consistency in the data collecting process, the pipe bend was divided into four sections along the 
curvature. These were labeled from Plane 1-5 including the ends of the bends. Figure 1 shows the planes in the order that they were sectioned, with an interval of $22.5^{\circ}$. All measurements refer to these planes in this order and position along the pipe bend.
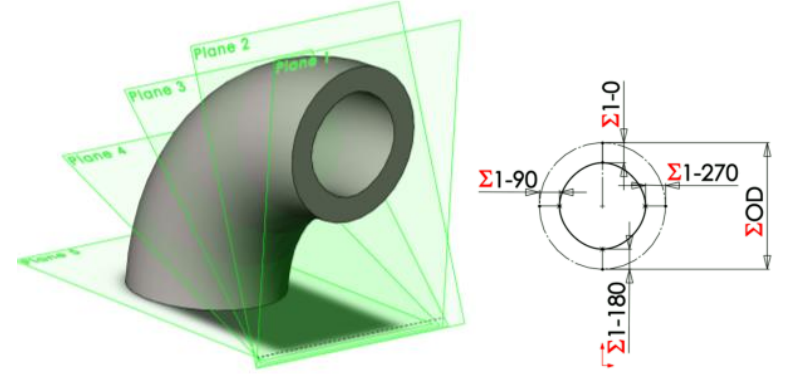

Figure 1. Measurement locations depicting the different planes along the pipe and the cross-section

At each plane, the cross-section was divided into many parts with fixed intervals to allow as many locations as needed. For the location of $0^{\circ}$, the extrados of the bend was chosen. This puts the intrados at $180^{\circ}$. Figure 2 shows the measurement locations and reading.

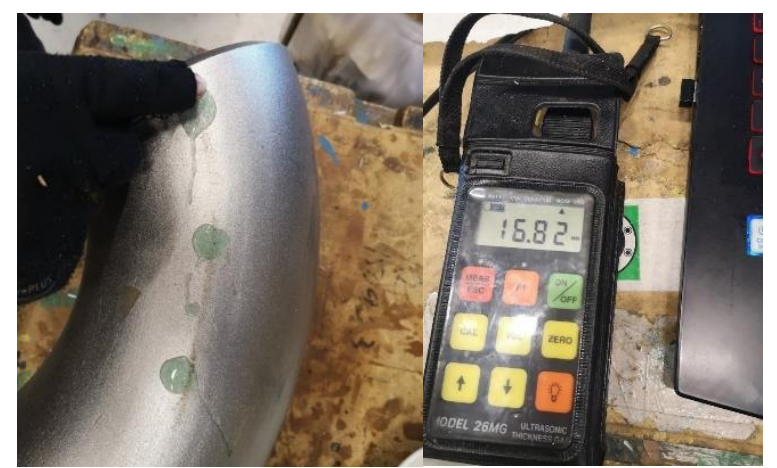

Figure 2. Measuring of 270 on plane 1 [left] and reading from NPS 6 SCH 80 Bend 1 [right]

\subsection{Measurement process}

Initially, extra measurements were taken on a single pipe bend, NPS 2 SCH 160, to create a large database of wall thickness. This included taking measurements at 45-degree increments for 8 locations per cross-section which gives a total 40 measurements for the bend. The number of measurements taken on each pipe was reduced after the NPS 2 was completed.

The data points were organized in Excel with each pipe size having its own sheet. The nominal wall thickness for an NPS $2 \mathrm{SCH} 160$ pipe is $8.378 \mathrm{~mm}$. Accounting for the minus Mill Tolerance, the wall thickness is then $7.646 \mathrm{~mm}$. In addition to wall thickness, using dial calipers the inner and outer diameter, and inner and outer bend radius were measured. Using the heat number on the stamp, details from the certified material test report were noted. A check for orality was performed on both pipe ends using dial calipers and noted if the measurement varied.

\section{Modeling}

The drawing programs SOLIDWORKS is used to make the geometry model of the component. The software program uses the centerline and cross-section profile to create a 3D model. The pipe model includes a perfectly circular outer diameter and a constant width for an ideal inner cross-section. To simulate the weakest allowed product that a manufacturer can produce, the NPS dimensions are used with the manufacturer's minus tolerance. This is specified in ASME B31.3 304.1.1.

\subsection{Finite Element Analysis (FEA)}

The SOLIDWORKS model was created using design table and global variables. The radius and outer diameter were set as global dimensions. The design table includes all the attributes and dimensions that are not defined as global from the configurations in Excel. The wall thickness was adjusted using the design table, which allowed for multiple model configurations.

Four split lines were added to the inner surface of the models at plane 2, plane 3 , plane 4 , and in the middle of the straight pipe as shown in Figure 3. The split lines allow for the simulation to extract results at the same location to ensure consistency in the evaluation.

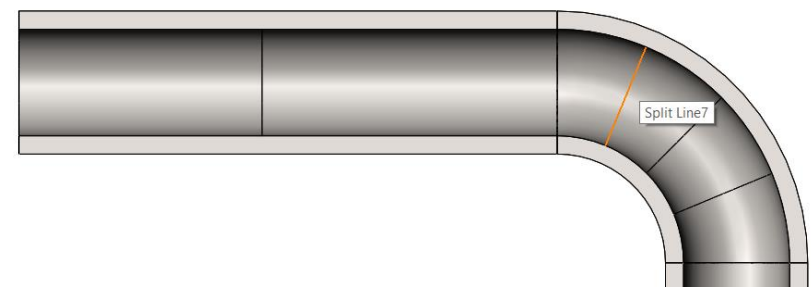

Figure 3. Split lines in the pipe model Simulation

Typically, a pipe is analyzed as a beam element (1D) with constant wall thickness. This creates a primary axis for movement and the other two are secondary directions. This model evaluates the complexity of the change in wall thickness of the pipe bend. The model was run as a solid (3D - tetrahedral element), which requires a larger mathematical model, and is not typically done for pipes.

\subsubsection{Boundary Conditions}

The straight pipes connecting the two ends of the bend were set as fixed at both ends. This avoids end cap effect as it restricts the movement on these surfaces completely. To reduce the concentrated stress from the fixed boundary on the attached straight pipe, various lengths were checked, and it was determined that the length of the pipe's bend radius was enough. In most cases 1.5 times bend radius was used for the straight pipe length. Figure 4 shows the fixed surfaces at the boundaries. 


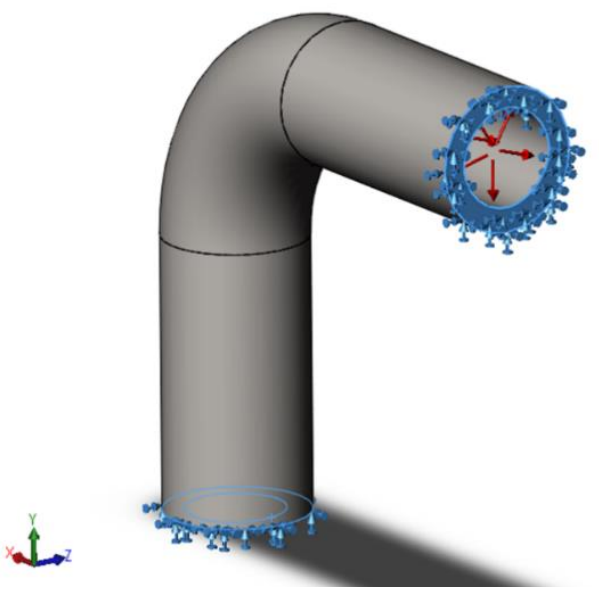

Figure 4. Pipe boundary conditions: fixed at both ends

\subsubsection{Pressure}

The internal pressure applied to all internal surfaces was determined using ASME B31.3 304.1.2 eq. (3a) (Engineers, ASME B31.3, 2002)

$$
t=\frac{P D}{2(S E+\gamma P)}
$$

By solving for pressure, $\mathrm{P}$, the internal pressure is determined. This is calculated with an allowable yield strength of $440 \mathrm{MPa}$ (S, UNS 32760 (SA-790) ASME BPVC II. D. M.-2015), outer diameter of $60.3 \mathrm{~mm}$ (D), wall thickness minus mill tolerance 7.6458 (t), coefficient for $\mathrm{t}>\mathrm{D} / 6$ and ferric steel temperature lower than $482{ }^{\circ} \mathrm{C}$ of $0.4(\gamma$, table 304.1 .1$)$, quality factor of 1 (E, table A-1B, spec no. A 790 material). For an NPS 2 $\mathrm{SCH} 160$ pipe, the maximum pressure is $124.18 \mathrm{MPa}$.

\subsubsection{Meshing}

The pipe, NPS 2 SCH 160 was evaluated with a variety of mesh sizes. Using the split lines shown in Figure 3, results were compared for 4 different mesh sizes (15 $\mathrm{mm}, 8 \mathrm{~mm}, 2.5 \mathrm{~mm}, 1 \mathrm{~mm})$. Figure 5 shows a comparison of the various mesh sizes. The medium(2.5 $\mathrm{mm}$ ) mesh was used in further cases.

Comparison of mesh sizing for NPS 2 SCH 160 Bend 1 at plane 3

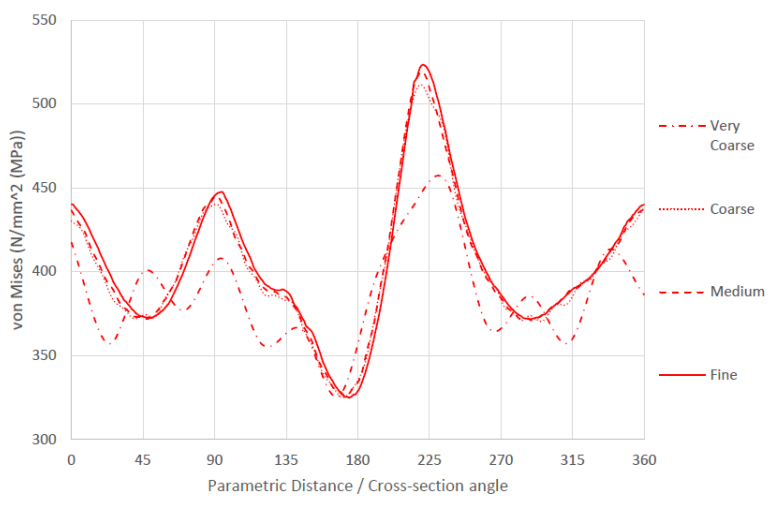

Figure 5. Probe results of plane 3 with variations on element sizes

\section{Results and discussion}

\subsection{Measurement results}

\subsubsection{Results for NPS 2 SCH160 bends}

For NPS 2 SCH 160, 10 bends were measured at 40 points. This gives a total of 400 measurements. Table 1 shows the measurement results for wall thickness at $90^{\circ}$ increments at the cross-section and Table 2 shows the results of the measurements at the midpoint of a crosssection.

Table 1. Wall thickness at $90^{\circ}$ increments

\begin{tabular}{|l|c|c|c|c|c|c|}
\hline \multirow{4}{*}{ Bend } & \multicolumn{5}{|c|}{ Wall thickness (mm) } \\
\cline { 2 - 7 } NPS 2 \\
\cline { 2 - 7 } SCH160 & & Plane 1 & Plane 2 & Plane 3 & Plane 4 & Plane 5 \\
\cline { 2 - 7 } & 90 & 9.50 & 8.25 & 8.15 & 8.20 & 8.47 \\
\cline { 2 - 7 } & 180 & 10.24 & 12.31 & 12.50 & 11.88 & 11.11 \\
\cline { 2 - 7 } & 270 & 9.67 & 9.43 & 9.18 & 9.47 & 9.57
\end{tabular}

Table 2. Wall thickness at midpoints of the $90^{\circ}$ increments at the cross section

\begin{tabular}{|c|c|c|c|c|c|c|}
\hline \multirow{6}{*}{$\begin{array}{c}\text { Bend } \\
\text { NPS } 2 \\
\text { SCH160 }\end{array}$} & \multicolumn{6}{|c|}{ Wall thickness (mm) - Midpoints } \\
\hline & \multicolumn{4}{|c|}{\begin{tabular}{|l|l|l|l} 
Plane 1 & Plane 2 Plane?
\end{tabular}} & \multicolumn{2}{|c|}{\begin{tabular}{|l|l|} 
Plane 4 Plane 5 \\
\end{tabular}} \\
\hline & 45 & 9.40 & 8.44 & 8.33 & 8.61 & 8.95 \\
\hline & 135 & 9.97 & 10.28 & 10.20 & 9.70 & 9.63 \\
\hline & 225 & 10.80 & 11.34 & 11.20 & 11.06 & 10.65 \\
\hline & 315 & 9.16 & 8.94 & 8.80 & 8.80 & 9.12 \\
\hline
\end{tabular}

In addition to wall thickness, using dial calipers the inner and outer diameter, and bend radius were measured which is shown in Table 3. Using the heat number on the stamp, details from the certified material test report were noted and presented in Table 4.

Table 3. Data collected for an NPS 2 SCH 160 bend: general dimension control

\begin{tabular}{|c|c|c|c|}
\hline \multicolumn{4}{|c|}{ Dimension control (mm) } \\
\hline & Plane 1 & Plane 3 & Plane 5 \\
\hline ID & 42.40 & N/A & 42.00 \\
\hline OD & 61.95 & 59.00 & 62.00 \\
\hline$R_{i}$ & 44.10 & N/A & 44.00 \\
\hline$R_{\mathrm{o}}$ & 105.00 & 104.00 & 107.00 \\
\hline
\end{tabular}

Table 4. NPS 2 SCH 160 bend: material test

\begin{tabular}{|l|c|l|c|}
\hline \multicolumn{3}{|l|}{ CERTIFIED MATERIAL TEST REPORT } \\
\hline Manufacturer & Shulz & Material & SA790 \\
\hline Cr & 25.4 & Standard & ASTM 790 \\
\hline Yield $0,2 \%(\mathrm{MPa})$ & 576.0 & & \\
\hline Yield $1 \%(\mathrm{MPa})$ & 669.0 & Origin & Spain \\
\hline Tensile $(\mathrm{MPa})$ & 870.0 & Manufactured & Germany \\
\hline
\end{tabular}


The measurements were analyzed using a sample standard deviation calculation. For a confidence of $95 \%$, the Z-value used was 1.96. Assuming the pipe to be uniform in thickness, all measurements were weighted equally. This gives an average of $9.53 \mathrm{~mm}, 95 \% \mathrm{CI}$ [9.44 mm, 9.61mm]. Figure 6 shows a normal distribution curve of NPS 2 SCH 160.

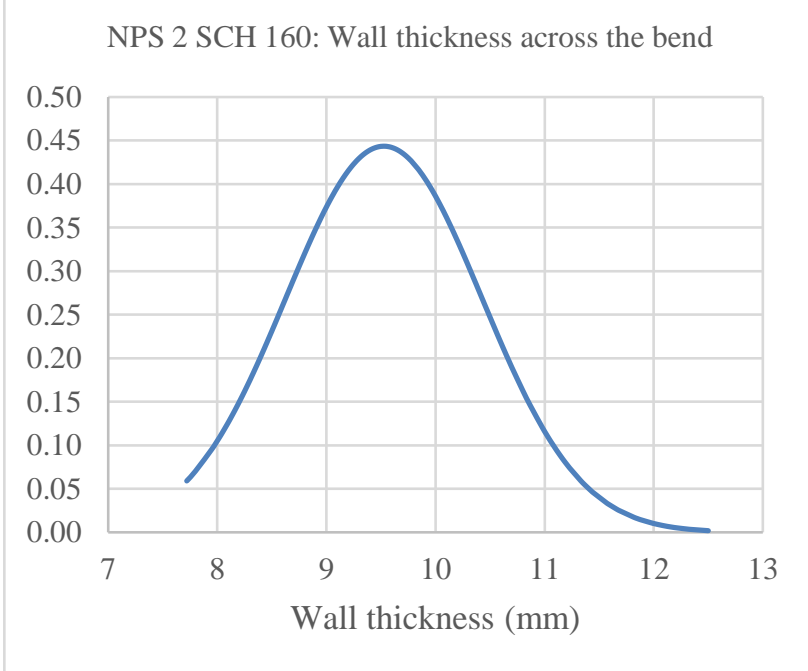

Figure 6. NPS 2 SCH 160, all measurements

There is a general trend of thinning along the extrados and thickening along the intrados. These three locations of wall thickness were the focus of the initial measuring process: end of pipe (plane 1 and plane 5), intrados (180/plane 3), and extrados (0/plane 3). These measurements were isolated and statistically evaluated to see the variation against the nominal wall thickness as shown in Figure 7.

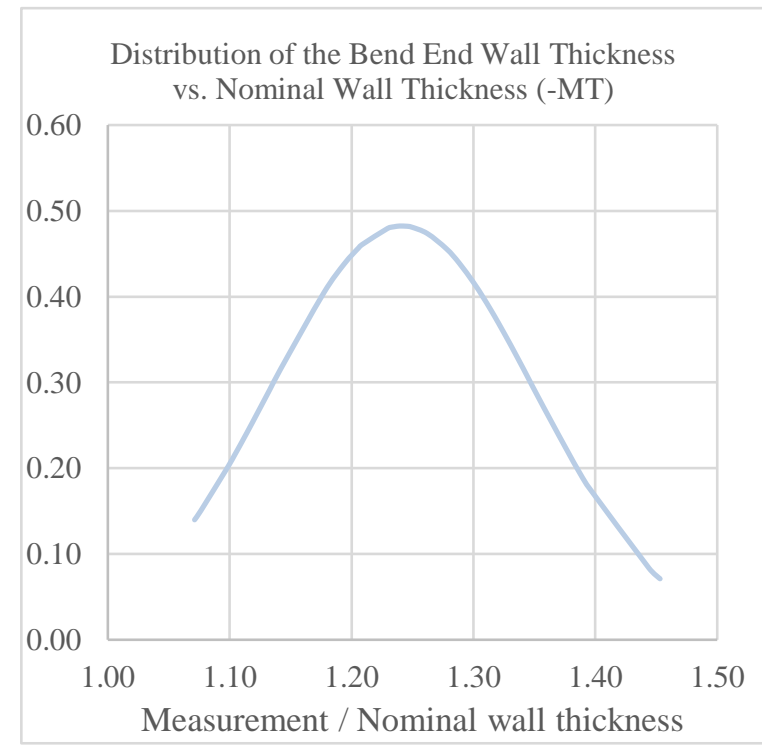

Figure 7. NPS 2 SCH 160 standard deviation diagram for the end wall thickness

\subsubsection{Measurement results for the other pipe sizes}

In total, 8 sizes of pipe bends were measured. Table 5 and Table 6 give an overview of all measurements.
Table 5. Quantity of pipes measured at $\mathrm{SFF}^{1}$

\begin{tabular}{|c|c|c|c|c|c|}
\hline & NPS 2 & NPS 3 & NPS 4 & NPS 6 & NPS 8 \\
\hline SCH 80 & 7 & & 7 & 10 & \\
\hline SCH 120 & & & & & 6 \\
\hline SCH 160 & 10 & 19 & 14 & 8 & \\
\hline
\end{tabular}

Table 6. Confidence interval of the pipe measurements ${ }^{2}$

\begin{tabular}{|c|c|c|c|}
\hline Size & Intrados & Extrados & End \\
\hline NPS & $11.86 \mathrm{~mm}$, & $8.42 \mathrm{~mm}$, & $9.47 \mathrm{~mm}$, \\
2 & $95 \% \mathrm{CI}$ & $95 \% \mathrm{CI}$ & $95 \% \mathrm{CI}$ \\
SCH & {$[11.60 \mathrm{~mm}$,} & {$[8.15 \mathrm{~mm}$,} & {$[9.38 \mathrm{~mm}$,} \\
160 & $12.12 \mathrm{~mm}]$ & $8.70 \mathrm{~mm}]$ & $9.56 \mathrm{~mm}]$ \\
\hline NPS & $8.08 \mathrm{~mm}$, & $5.40 \mathrm{~mm}$, & $6.76 \mathrm{~mm}$, \\
2 & $95 \% \mathrm{CI}$ & $95 \% \mathrm{CI}$ & $95 \% \mathrm{CI}$ \\
SCH & {$[7.74 \mathrm{~mm}$,} & {$[5.19 \mathrm{~mm}$,} & {$[6.48 \mathrm{~mm}$,} \\
80 & $8.41 \mathrm{~mm}]$ & $5.61 \mathrm{~mm}]$ & $7.05 \mathrm{~mm}]$ \\
\hline NPS & $15.60 \mathrm{~mm}$, & $11.79 \mathrm{~mm}$, & $13.59 \mathrm{~mm}$, \\
3 & $95 \% \mathrm{CI}$ & $95 \% \mathrm{CI}$ & $95 \% \mathrm{CI}$ \\
SCH & {$[15.23 \mathrm{~mm}$,} & {$[11.37 \mathrm{~mm}$,} & {$[13.4 \mathrm{~mm}$,} \\
160 & $15.98 \mathrm{~mm}]$ & $12.21 \mathrm{~mm}]$ & $13.79 \mathrm{~mm}]$ \\
\hline $\mathrm{NPS}$ & $17.79 \mathrm{~mm}$, & $12.90 \mathrm{~mm}$, & $15.14 \mathrm{~mm}$, \\
4 & $95 \% \mathrm{CI}$ & $95 \% \mathrm{CI}$ & $95 \% \mathrm{CI}$ \\
$\mathrm{SCH}$ & {$[17.55 \mathrm{~mm}$,} & {$[12.69 \mathrm{~mm}$,} & {$[14.99 \mathrm{~mm}$,} \\
160 & $18.03 \mathrm{~mm}]$ & $13.11 \mathrm{~mm}]$ & $15.29 \mathrm{~mm}]$ \\
\hline $\mathrm{NPS}$ & $10.43 \mathrm{~mm}$, & $7.95 \mathrm{~mm}$, & $9.17 \mathrm{~mm}$, \\
4 & $95 \% \mathrm{CI}$ & $95 \% \mathrm{CI}$ & $95 \% \mathrm{CI}$ \\
SCH & {$[10.15 \mathrm{~mm}$,} & {$[7.83 \mathrm{~mm}$,} & {$[9.03 \mathrm{~mm}$,} \\
80 & $10.71 \mathrm{~mm}]$ & $8.08 \mathrm{~mm}]$ & $9.30 \mathrm{~mm}]$ \\
\hline $\mathrm{NPS}$ & $23.47 \mathrm{~mm}$, & $18.44 \mathrm{~mm}$, & $20.72 \mathrm{~mm}$, \\
6 & $95 \% \mathrm{CI}$ & $95 \% \mathrm{CI}$ & $95 \% \mathrm{CI}$ \\
SCH & {$[23.21 \mathrm{~mm}$,} & {$[18.17 \mathrm{~mm}$,} & {$[20.56 \mathrm{~mm}$,} \\
160 & $23.72 \mathrm{~mm}]$ & $18.70 \mathrm{~mm}]$ & $20.88 \mathrm{~mm}]$ \\
\hline $\mathrm{NPS}$ & $18.46 \mathrm{~mm}$, & $14.53 \mathrm{~mm}$, & $16.50 \mathrm{~mm}$, \\
6 & $95 \% \mathrm{CI}$ & $95 \% \mathrm{CI}$ & $95 \% \mathrm{CI}$ \\
$\mathrm{SCH}$ & {$[18.19 \mathrm{~mm}$,} & {$[14.41 \mathrm{~mm}$,} & {$[16.32 \mathrm{~mm}$,} \\
80 & $18.72 \mathrm{~mm}]$ & $14.65 \mathrm{~mm}]$ & $16.68 \mathrm{~mm}]$ \\
\hline $\mathrm{NPS}$ & $22.66 \mathrm{~mm}$, & $17.16 \mathrm{~mm}$, & $20.10 \mathrm{~mm}$, \\
8 & $95 \% \mathrm{CI}$ & $95 \% \mathrm{CI}$ & $95 \% \mathrm{CI}$ \\
$\mathrm{SCH}$ & {$[22.44 \mathrm{~mm}$,} & {$[16.96 \mathrm{~mm}$,} & {$[19.9 \mathrm{~mm}$,} \\
120 & $22.89 \mathrm{~mm}]$ & $17.36 \mathrm{~mm}]$ & $20.29 \mathrm{~mm}]$ \\
\hline
\end{tabular}

To compare the variation, all thicknesses were scaled against their respective nominal wall thickness. This creates a baseline of 1 to compare all pipes simultaneously in a single graph, where 1 represents the measured wall thickness equal to the nominal wall thickness minus MT. Figure 8(intrados), Figure 9(extrados), and Figure 10(end) all have the same trends of clumping of the normal distribution curves with an outlier being the NPS $6 \mathrm{SCH} 80$. There is a large spread in some of the sizes, NPS 3 SCH 160, for example. This had 19 total bends, so it had the largest sample size. Three different manufacturers were included in this sampling and 4 different heat numbers. Most of the bends measured had only one manufacturer and one heat number.

\footnotetext{
${ }^{1}$ NPS 8 SCH 120 is a seam welded pipe

2 The bend end has four points for each bend as shown in Table 6 . These are at plane 1 and plane 5 at $0^{\circ}$ and $180^{\circ}$.
} 


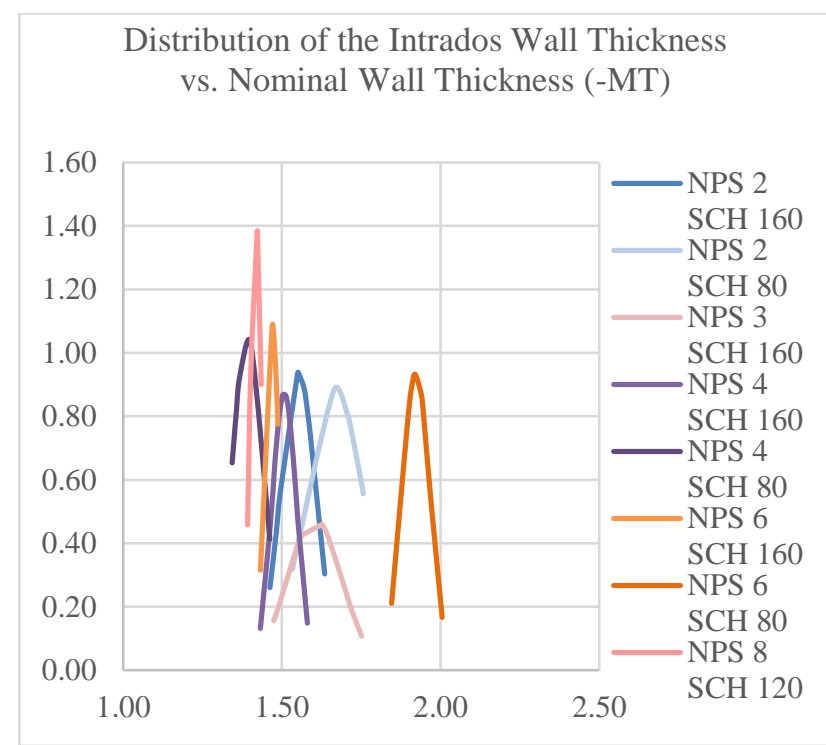

Figure 8. Distribution of the Intrados Wall Thickness / Nominal Wall Thickness (less mill tolerance)

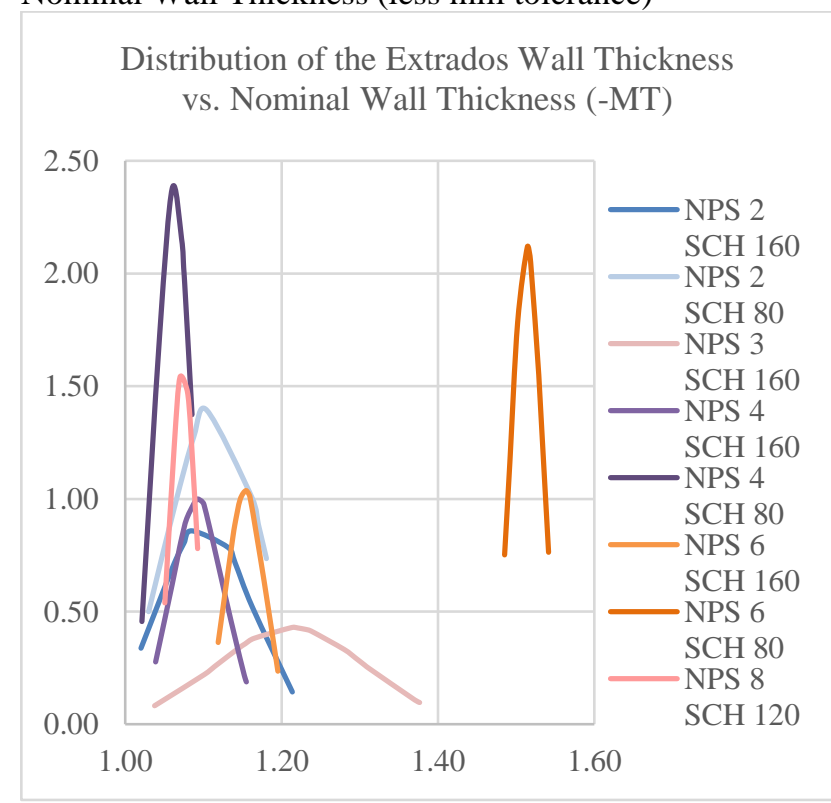

Figure 9. Distribution of the Extrados Wall Thickness / Nominal Wall Thickness (less mill tolerance)

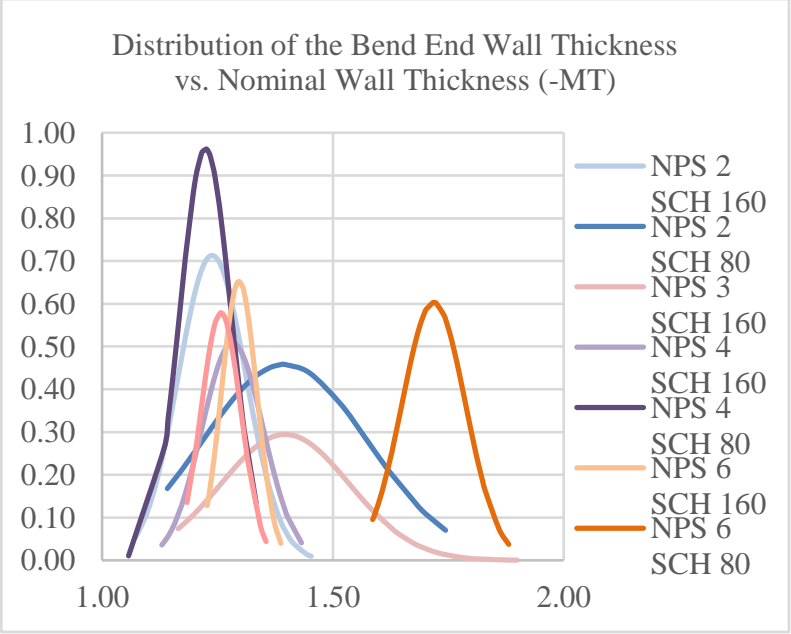

Figure 10. Distribution of the End Wall Thickness / Nominal Wall Thickness (less mill tolerance)
While mill tolerances are removed for comparison for a stress analysis, the standard pipe bends may be out of allowable tolerances due to total weight. Further research/observations are necessary as well as verification of the relevant standard for Super Duplex.

This article does not verify if the implication that the bends that were measured could be over the allowable limits due to the increased wall thickness. This can affect the total weight of the system and therefore support calculations and stress analysis. The NPS 6 pipe had nearly $50 \%$ more material. This is an outlier for the data set, but it should be noted that the pipe measurements included various sizes from the same manufacturer as the outlier size.

\subsection{Simulation results}

\subsubsection{Comparison of quantity of measurements per bend}

Two measurements were analyzed to evaluate critical aspects of the pipe bend when modeled and pressure is applied. This article compares the NPS 2 SCH 160 bend with 40 measurements and 20 measurements. The difference being the number of measurements per crosssection is shown in Figure 11.

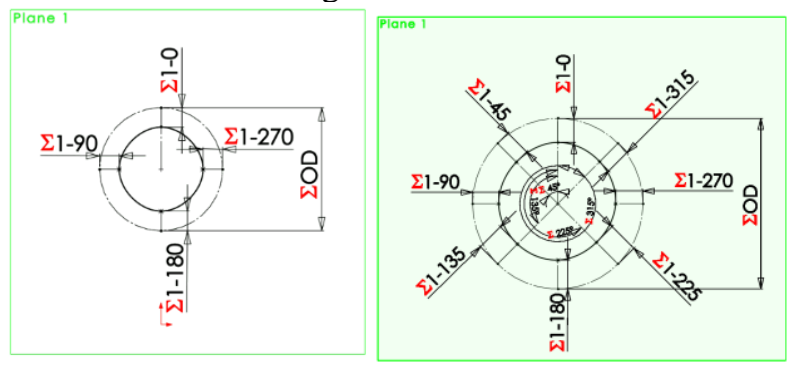

Figure 11. Cross section of plane on model $1.90^{\circ}$ increments for 20 in total [left] and $45^{\circ}$ increments for 40 in total [right]

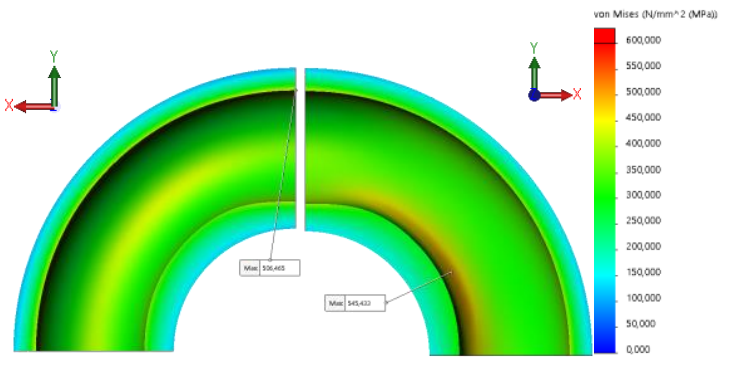

Figure 12. Stress of the pipe with 20 measurements [left] and 40 measurements [right]

Figure 12. shows the stress plot of NPS 2 SCH 160 bend. A transition of geometry, for example from a bend to a straight line, can have a stress concentration as seen in the result at the top of the bend on the pipe with 20 measurements, left in Figure 12. The split lines are utilized for a numerical comparison between the two results. 


\subsubsection{Comparison of measured models to standard pipe bends}

The split line added in the middle of the attached straight pipe is used to check the strength of the standard straight pipe. The allowable minus mill tolerances are used in this analysis because all measured pipe bends were finished fabricated and the maximum stress the thinnest allowed straight pipe wall could tolerate is used as a baseline.

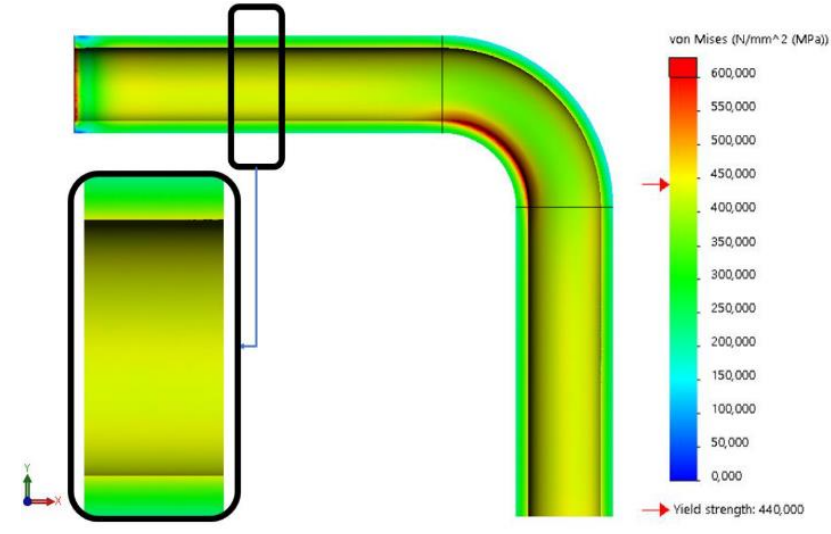

Figure 13. Straight pipe with constant wall thickness stress plot

Figure 13 shows the pipe stress from the applied internal pressure. The peak stress on the split line is $490 \mathrm{MPa}$. This was plotted as a baseline (black) in Figure 14.

Note that the peak stress found from the model of 20 data points, at $484 \mathrm{MPa}$ is lower than the straight pipe results, $490 \mathrm{MPa}$.

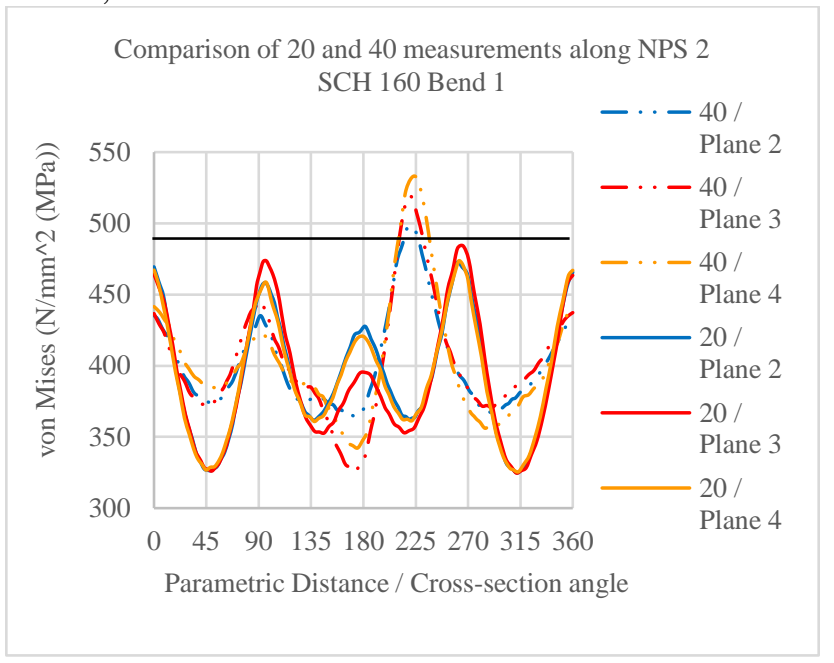

Figure 14. Varying quantity of measurements along a bend

The highest stress from the model with 20 measurements has peaks around $90^{\circ}$ and $270^{\circ}$ in

Figure 14. The peak stress is $484 \mathrm{MPa}$ on plane 3 at about $270^{\circ}$. This correlates to the yellow area in Figure 12. The highest stress from the model with 40 measurements has peaks around $225^{\circ}$ in

Figure 14. The peak stress from the three split lines is on plane 4 at about $225^{\circ}$ with $532 \mathrm{MPa}$. The peak stress on plane 3 is $519 \mathrm{MPa}$ and plane 2 is $498 \mathrm{MPa}$, also at about $225^{\circ}$. This correlates to the orange area in Figure 12.

The results of the highest stress within the bend (intrados) is compared with its respective straight pipe with the same pressure applied. To allow for comparison of all measured pipes, the results are in the form of a percent of the stress and wall thickness of a nominal straight pipe. The chart in Figure 15 shows the different measured pipe sizes. The vertical axis gives a comparison of the pipe bends stress and its straight pipe counterpart. The nominal pipe sizes that stay below the $100 \%$ line indicates that the bend has less stress than the straight pipe. The ones that are placed above the line contains more stress and are therefore weaker than the straight pipe. The horizontal axis marks the percentage of the measured wall thickness divided by the nominal pipe size i.e. the NPS $3 \mathrm{SCH} 160$ that is $65-73 \%$ thicker.

The NPS $4 \mathrm{SCH} 80$ has a higher stress than the straight pipe. This can be an issue because a pipe component, bend in this case, is supposed to be stronger than its straight pipe counterpart. Figure 15 shows that the thickness for this pipe size has its curve farthest left, implying thinner wall thickness than other sizes.

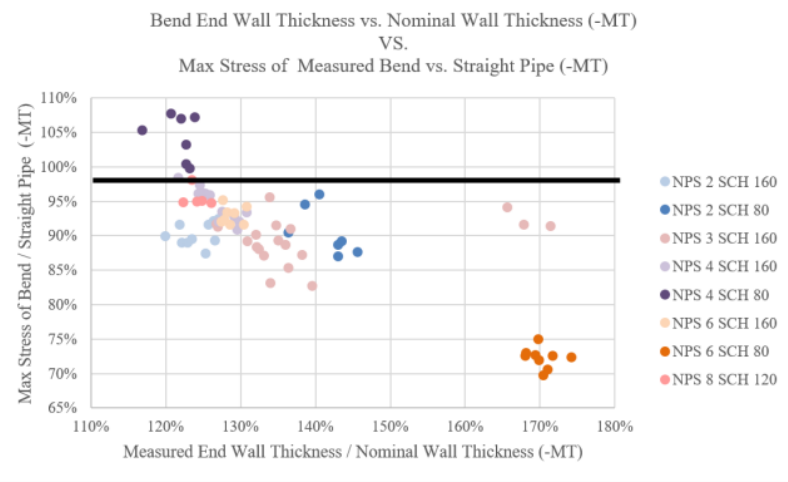

Figure 15. FEA Stress Results of the Bend End: A Comparison between the measured thickness and nominal thickness (-MT)

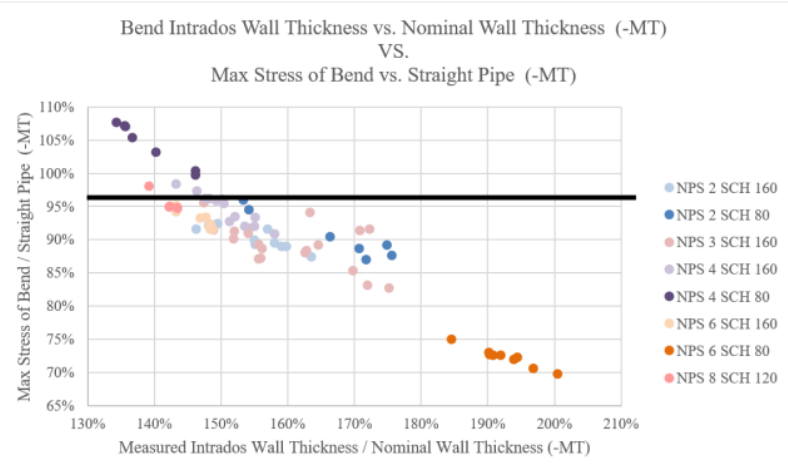

Figure 16. FEA Stress Results of the Intrados: A Comparison between the measured thickness and nominal thickness (-MT) 


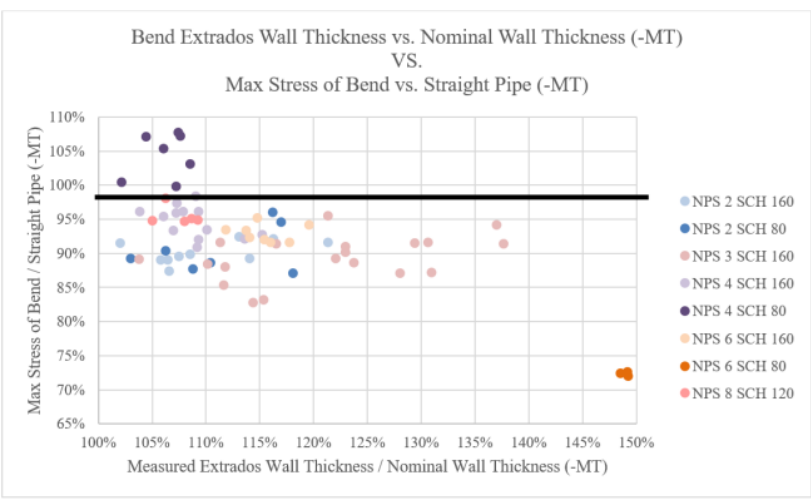

Figure 17. FEA Stress Results of the Extrados: A Comparison between the measured thickness and nominal thickness (-MT)

As shown in Figure 15, Figure 16, and Figure 17, there is a random spread in the different sizes. In Figure 16, an even curve is clearly formed which indicates a relation between the thickness of the intrados and the nominal pipe size when pressure is applied. The warehouse at SFF did not have any duplex bends in NPS $3 \mathrm{SCH} 80$, so 316 was also measured to include the size. Figure 18. shows the results of all measured bends, so there is a blend of materials. The linear trend line for the results of the intrados has an $\mathrm{R}^{2}$ value of 0.8281 .

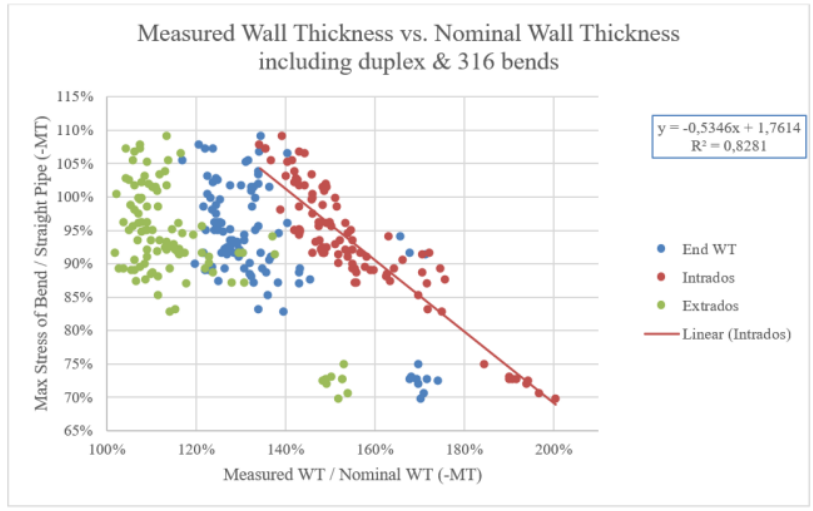

Figure 18. Result comparison of all three locations.

Calibration of the ultrasonic device in between each size, Temperature difference for the material expanding and contracting though this is minor, the material and resistance against rays, curvature which made measuring difficult and the use of zalo are all variables that may have had an effect on the results in this article.

\subsection{Analyzing the Measurements}

Early in this project, it was very clear the pipe bends do not have a uniform thickness. A simplification on the measurement locations, like the use of symmetry, would not accurately represent any given location between the bend end to the intrados/extrados.

There was a significant spread of how much thicker the bend was in comparison to the nominal pipe standard. This was especially noticeable in the samples that were of the same size but had multiple heat numbers and manufacturers.
In general, the results do not deviate from the expectations, but some variations were noticed. The NPS 4 SCH 80 is the one component size that is not in correspondence with the ASME B16.9 Standard based on the 6 point measurement. Detailed models of the NPS $4 \mathrm{SCH} 80$ could allow for better evaluation of the pipe. The simplified analysis of this pipe size is the only case where the bend was weaker than the corresponding straight pipe, which does not confirm ASME B16.9 Section 2.1. It is worth noting that the manufacturer of this particular batch is the same as the one with excessive wall thickness on the NPS 6 SCH 80.

With regards to the FEA model comparisons, the 40 point measurement shows a peak stress of over $500 \mathrm{MPa}$ whereas the 20 point measurement is under the peak stress at $484 \mathrm{MPa}$. This implies a loss in between the modeling that is not covered by a standard spline fit in SOLIDWORKS model.

\section{Conclusion}

Based on the statistical analysis of the 81 measured bends, the lower bound values of the intrados with a 99.9\% CI is $52.1 \%$ larger than the nominal wall thickness (-MT). The extrados is $12.4 \%$ larger than the nominal wall thickness (-MT), or nearly equal to the nominal wall thickness.

The database is still incomplete and should be expanded upon to be able to recognize any trends and map critical dimensions. There are 2 sizes (NPS 4 SCH 80 \& NPS 6 SCH 80) that should be further investigated. There is insufficient data to confirm section 2.1 of ASME B16.9.

The most important comparison is the physical pressure test with the mathematical model which will verify if the modeling is good enough for further use, or if the technique needs adjustments.

The application of pipe bends in the subsea industry is crucial components and if they aren't close enough it can lead to costly consequences. Further research into the critical dimensions and deviation within the industry is recommended.

\section{References}

American Society of Mechanical Engineers. ASME Code for Process Piping, ASME B31.3. 2002.

American Society of Mechanical Engineers. Pipe Flanges and Flanged Fittings, ASME B16.9. 2012.

Det Norske Varitas. DNVGL-RP-F112.DNV, 2018.

Inspectioneering. Overview of Hydrogen Induced Cracking (HIC). 2020 [Online], Available: https://inspectioneering.com. [Accessed 20 April 2020] 